\title{
The effect of intravitreal ranibizumab injection on visual acuity and central macular thickness in subgroups with neovascular age-related macular degeneration
}

\author{
Ebru Cömert ${ }^{a *}$, Hakkı Birinci ${ }^{\mathrm{b}}$ Yüksel Süllü̈ \\ ${ }^{a}$ Department of Ophthalmology, Faculty of Medicine, Ondokuz Mayls University, Samsun, Turkey \\ ${ }^{b}$ Department of Ophthalmology, Medical Park Hospital, Samsun, Turkey
}

\section{ARTICLE INFO}

\section{Article History}

Received $\quad 29 / 08 / 2012$

Accepted $\quad 15 / 09 / 2012$

\section{* Correspondence to:}

Ebru Cömert

Department of Ophthalmology,

Faculty of Medicine,

Ondokuz Mayıs University,

Samsun, Turkey

e-mail: dr.ebrucomert@gmail.com

\section{Keywords:}

Age related macular degeneration

Central macular thickness

Choroidal neovascularization

Ranibizumab

Visual acuity

\author{
ABSTRACT
}

In this study, we assessed the effect of at least three intraocular ranibizumab injections, which were performed for the treatment of subfoveal choroidal neovascularization (CNV) secondary to neovascular age related macular degeneration (AMD), on best corrected visual acuity (VA) and central macular thickness (CMT) in 89 patients who were classified by the component of lesion. Flouroscein angiography was performed and CNV have been classified in classic, occult or mixt (classic+occult) type. Best corrected VA was measured by Snellen card and was recorded in logMAR-logarithm of the minimum angle of resolution values. CMT was measured by optical coherence tomography. While CMT decreased significantly in patients with classic, mixt or occult type lesion ( $p=0.000$; $0.011 ; 0.004$, respectively), the increase in VA was detected only in patients with classic type lesion $(\mathrm{p}=0.001)$. A positive significant correlation between the decrease in CMT and the decrease in VA $(\log$ MAR) was reported only in the patients with occult type lesion ( $p=0.03$ ). In conclusion, the anatomical improvement, that is seen as a result of the treatment of AMD with intravitreal ranibizumab injection, is not associated with the increase in visual acuity in all types of CNV.

J. Exp. Clin. Med., 2013; 30:1-3

\section{Introduction}

Age-related macular degeneration (AMD) is the most common cause of legal blindness of 65 and over age group. Neovasculer AMD forms the 10 percent of the AMD but is responsible for 90 percent of the legal blindness (Ferris et al., 1984; Bird et al., 1995).

In most of the cases, choroidal neovascularization (CNV) is located under the fovea (subfoveal) (Arias et al., 2009). At present, there are alternative treatment methods for subfoveal CNV such as photodynamic treatment (PDT) with verteporfin, intravitreal steroid or anti vascular endothelial growth factors (anti-VEGF; macugen, bevacizumab or ranibizumab) injection.

Ranibizumab (Lucentis, Genentech Inc. South San Francisco, CA) is a recombinant, humanized monoclonal antibody fragment that inhibits all VEGF-A isoforms and was approved by the United States Food and Drug Administration for the treatment of neovascular AMD in June 2006 (Parravano et al., 2010).
In our study, we assessed the effects of at least three intraocular ranibizumab injections, performed for the treatment of subfoveal choroidal neovascularization, on best corrected visual acuity and central macular thickness in patients who were classified by the component of lesion.

\section{Materials and methods}

We reviewed retrospectively, the cards of patients with neovascular AMD treated with intravitreal ranibizumab injections at Ondokuz Mayis University, Department of Ophthalmology, from Feb 2009 to June 2011. We included 89 eyes of 89 patients, who underwent three consecutive injections of ranibizumab at monthly intervals.

At baseline, best corrected visual acuity (VA) was measured by Snellen card and was recorded in logMAR-logarithm of the minimum angle of resolution values. Flouroscein anjiography (FA, Carl Zeiss Meditec AG, Jena, Germany) was performed and CNV have been classified in classic, occult or mixt (classic+occult) type. Central macular thickness (CMT) 
was measured by optical coherence tomography (OCT, Stratus OCT, Carl Zeiss Meditec, Jena, Germany). The patients were subclassified according to the CNV type.

Intravitreal ranibizumab injections were performed in the office under sterile conditions. After topical anesthesia, the lids and ocular surface were disinfected with $5 \%$ povidone iodine. An eye speculum was placed in the eye. Intravitreal injections of $0.5 \mathrm{mg} / 0.05 \mathrm{~mL}$ ranibizumab were performed $4 \mathrm{~mm}$ behind the limbus in the temporal quadrant with a $27-$ gauge needle. Antibiotic ointment was placed on the eye after the injection. Topical drops of levofloxacin were prescribed 4 times a day for one week. Injections were repeated at first and second months.

After three consecutive injections of ranibizumab, the decision of new injection was given if there was angiographic leakage or subretinal fluid on OCT scans. The number of injections, follow-up time, VA and CMT at last visit were recorded. Main end points were the change in VA, CMT and the correlation between the changes in VA and CMT.

Statistical analysis was performed using SPSS 15.0. Visual acuity was converted to $\log$ MAR before analysis. Normality tests were applied to all measurement variables. Wilcoxon test was used for non-normally distributed variables to compare the means before and after treatment. Correlation between change in VA and CMT was tested using the Spearman's Correlation Test. $\mathrm{P}<0.05$ was considered statistically significant.

\section{Result}

Total of 89 eyes of 89 patients were included in the study. Patients were subclassified in three groups according to the lession type. The groups were similar in age, baseline VA and CMT. Descriptive data were given in Table 1 . There were no differences between groups in age, baseline VA and baseline CMT ( $>>0.05)$.

\section{Table 1. Descriptive data of the subgroups}

\begin{tabular}{lccc} 
& Classic type & Mixt type & Occult type \\
\hline Number of patients (n, \%) & $43(48.3 \%)$ & $9(10.1 \%)$ & $37(41.6 \%)$ \\
Age (year) & $72(51-83)$ & $74(51-82)$ & $73(52-85)$ \\
$\quad$ Female (n, \%) & $16(37.2 \%)$ & $4(44.4 \%)$ & $21(56.8 \%)$ \\
$\quad$ Male (n, \%) & $27(62.8 \%)$ & $5(55.6 \%)$ & $16(43.2 \%)$ \\
Baseline VA (logMAR) & $1(0.2-2.3)$ & $0.7(0.4-1.3)$ & $1.0(0.1-2.0)$ \\
Baseline CMT $(\mu \mathrm{m})$ & $338(119-797)$ & $359(188-451)$ & $274(173-637)$ \\
\hline
\end{tabular}

VA; visual acuity, logMAR; logarithm of the minimum angle of resolution values, CMT; central macular thickness

When all of the patients were evaluated, we found that VA decreased from $0.97(0.1-2.3) \log$ MAR to $0.83(0.0-2.3)$ $\operatorname{logMAR}$ and CMT decreased from $319.3 \mu \mathrm{m}$ (119-797) to $253.9 \mu \mathrm{m}(92-571)$ significantly with treatment $(\mathrm{p}=0.005$;
$<0.001$, respectively). There was no correlation between the changes in VA and CMT $(\mathrm{p}=0.83)$.

In patients with classic type $\mathrm{CNV}$, median values of VA

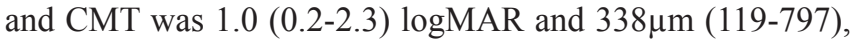
respectively, before treatment. The median number of injections was 3 (3-5) and patients were followed up for 7 (4-30) months (4-30). After treatment, the median values of VA and CMT was 0.7 (0.1-2.3) $\log$ MAR and 256 $\mu \mathrm{m}$ (92-571), respectively. The median values of VA and CMT change was $0.2(-1.0-1.1) \log$ MAR and $69.8 \mu \mathrm{m}(-187-512)$, respectively and the changes were significant. $(\mathrm{p}=0.001, \mathrm{p}=0.000$, respectively) (Table 2). There was no correlation between the changes of VA and CMT $(\mathrm{p}=0.82)$.

In patients with mixt type $\mathrm{CNV}$, median values of VA and CMT was 0.7 (0.4-1.3) $\log$ MAR and $359 \mu \mathrm{m}$ (188-451), respectively, before treatment. Three (3-4) intravitreal ranibizumab injections were applied and patients were followed up for 8 (4-19) months. After treatment, the median values of VA

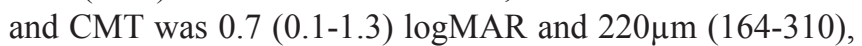
respectively. The median values of VA and CMT change was $0.03(-0.3-0.3) \log$ MAR and $101.9 \mu \mathrm{m}(-12-269)$, respectively. While there was a significant change in CMT $(p=0.011)$, VA did not change significantly after treatment $(\mathrm{p}=0.60)$ (Table 2 ). There was no correlation between the changes of VA and CMT $(p=0.30)$.

In patients with occult type $\mathrm{CNV}$, median value of VA and CMT was $1.0(0.1-2.0) \operatorname{logMAR}$ and $274 \mu \mathrm{m}$ (173-637), respectively, before treatment. The median number of injections was 3 (3-8) and patients were followed up for 7 (4-21) months. After treatment, the median value of VA and CMT was $0.7(0.0-2.3) \log$ MAR and $225 \mu \mathrm{m}$ (153-530), respectively. The median value of VA and CMT change were 0.07 (-1.6$0.8) \log$ MAR and $51.5 \mu \mathrm{m}(-341-459)$, respectively. While there was a significant change in CMT $(\mathrm{p}=0.004)$, VA did not change significantly after treatment $(\mathrm{p}=0.20)$ (Table 2$)$. There was a positive significant correlation between the changes of VA and CMT ( $\mathrm{p}=0.03, \mathrm{r}=0.35)$.

\section{Discussion \\ The results of this retrospective study showed that intravitreal injections of ranibizumab are associated with increase in vi- sual acuity and decrease in central macular thickness for the group as a whole. Subgroup analysis revealed that decrease in CMT was noted overall but VA increased only in the patients with classic type CNV. Gardner, Vujosevic and Parravano explained this finding by the duration of the macular disease (Gardner et al., 2002; Vujosevic et al., 2006; Parravano et al., 2010). They suggested that a prolonged intra-and/or sub- retinal edema could lead to persisting and irreversible dam- age to the photoreceptors that could influence the functional changes after treatment. Classic CNV leads to more rapid loss}

Table 2. The median, minimum, maximum values of visual acuity (logMAR) and central macular thickness( $\mu \mathrm{m})$ of the patients with classic, mixt or occult lesions before and after treatment

\begin{tabular}{|c|c|c|c|c|c|c|}
\hline & \multicolumn{2}{|c|}{ Visual acuity (logMAR) } & \multicolumn{4}{|c|}{ Central macular thickness $(\mu \mathrm{m})$} \\
\hline & Before treatment & After treatment & $p$ value & Before treatment & After treatment & $p$ value \\
\hline Classic type & $1(0.2-2.3)$ & $0.7(0.1-2.3)$ & 0.001 & 338 (119-797) & $256(92-571)$ & 0.000 \\
\hline Mixt type & $0.7(0.4-1.3)$ & $0.7(0.1-1.3)$ & 0.60 & $359(188-451)$ & $220(164-310)$ & 0.011 \\
\hline Occult type & $1(0.1-2.0)$ & $0,7(0.0-2.3)$ & 0.20 & $274(173-637)$ & $225(153-530)$ & 0.004 \\
\hline
\end{tabular}


of vision when compared with other types of neovascularization and be noticed sooner, the treatment begins earlier. As a result, more photoreceptors are protected. In contrary, Shin et al. (2011) suggested that classic CNV cause irreversible damage in the foveal photoreceptor layer more frequently than occult $\mathrm{CNV}$, because classic $\mathrm{CNV}$ occupies subretinal space more extensively than occult $\mathrm{CNV}$ and occult lesions remain beneath the retina pigment epithelium (RPE) but they did not find a significant difference in VA and distrupted IS/OS (inner segment/outer segment of photoreceptor) length changes between classic and occult CNV.

In our study, while there were significant improvements in VA and CMT, no correlation was detected between the change in VA and CMT in the group of all patients. When we evaluated the subgroups, there was no correlation between the change in VA and CMT in the groups of classic and mixt type $\mathrm{CNV}$, on the other hand there was a positive significant correlation in the occult CNV group ( $\mathrm{r}=0.35, \mathrm{p}=0.03)$. As a similar result, Bhatnagar et al. (2007) found that ranibizumab therapy was associated with significant improvements in mean visual acuity and central macular thickness for the group of all patients but the change in visual acuity showed only a very small correlation with the change in central macular thickness $(r=-0.17)$. Shin et al. (2011) found that preservation of the IS/ OS and external limiting membrane, thinner central macular thickness and shorter choroidal neovascularization height before treatment were associated with intact photoreceptor integrity after resolution of exudation. The findings of our study may be resulted from the difference of the subgroups in these parameters.
Some studies suggested that visual acuity is not useful alone to assess the functional improvement with ranibizumab treatment in AMD. Retinal sensitivity is another parameter that became the subject of the new researches Squirrell et al. (2010). Compared the observed change in best corrected VA with change in central retinal sensitivity pretreatment and one month after three consecutive treatments with ranibizumab in patients with neovascular AMD and they found that compared with microperimetry, VA seems to significantly underestimate the change in visual function experienced by patients treated with ranibizumab. Parravano et al. (2010) found that intravitreal injections of $0.5 \mathrm{mg}$ ranibizumab determine progressive improvement of retinal sensitivity until 24 months, suggesting that microperimetry may give additional information about macular function not given by visual acuity alone. In addition, changes of functional status, as expressed by mean retinal sensitivity and visual acuity, were not found to be related either to baseline status or to the observed CRT changes.

The limitations of our study are retrospective, nonrandomized native and small number of patients. Further investigation with a large number of patients and evaluation of associated factors, such as retinal sensitivity, IS/OS and external limiting membrane integrity, choroidal neovascularization height, should be performed.

As a result, the anatomical improvement, that is seen as a result of the treatment of AMD with intravitreal ranibizumab injection, is not associated with the increase in visual acuity in all types of CNV.

\section{REFERENCES}

Arias, L., Ruiz-Moreno, J., Gomez-Ulla, F., Fernandez, M., Montero J., 2009. 1-year retrospective review of ranibizumab for naive nonsubfoveal choroidal neovascularization secondary to age-related macular degeneration. Retina. 29, 1444-1449.

Bhatnagar, P., Spaide, R.F., Takahashı, B.S., Peragallo, J.H., Freund, K.B., Klancnik, Jr.J.M., Cooney, M.J., Slakter, J.S., Sorenson, J.A., Yannuzzi, L.A., 2007. Ranibizumab for treatment of choroidal neovascularization secondary to age-related macular degeneration. Retina. 27, 846-850.

Bird, A.C., Bressler, N.M., Bressler, S.B., Chisholm, I.H., Coscas, G., Davis, M.D., de Jong, P.T., Klaver, C.C., Klein, B.E., Klein, R., 1995. An international classification and grading system for age-related maculopathy and age-related macular degeneration. Surv. Ophthalmol. 39, 367-374.

Ferris, F.L., Fine, S.L., Hyman, L., 1984. Age-related macular degeneration and blindness due to neovascular maculopathy. Arch. OphthalmolChic. 102, 1640-1642.

Gardner, T.W., Antonetti, D.A., Barber, A.J., LaNoue, K.F., Levison, S.W., 2002. Diabetic retinopathy: More than meets the eye. Surv. Ophthalmol. 47, 252-253.

Parravano, M., Francesco, O., Tedesch1, M., Chiaravallot1, A., Perıllo, L., Boccassını, B., Varano, M., 2010. Retinal functional changes measured by microperimetry in neovascular age-related macular degeneration treated with ranibizumab. Retina. 30, 1017-1024.

Shin, H.J., Chung, H., Kım, H.C., 2011. Association between foveal microstructure and visual outcome in age-related macular degeneration. Retina. 31, 1627-1636.

Squirrell, D.M., Mawer, N.P., Mody, C.H., Brand, C.S., 2010. Visual outcome after intravitreal ranibizumab for wet age-related macular degeneration. Retina. 30, 436-442.

Vujosevic, S., Midena, E., Pilotto, E., Radin, P.P., Chiesa, L., Cavar-zeran, F., 2006. Diabetic macular edema: Correlation between micro perimetry and optical coherence tomography findings. Invest Ophth. Vis. Sci. 47, 3044-3051. 